\title{
ON THE WEAK SOLUTION OF A THREE-POINT BOUNDARY VALUE PROBLEM FOR A CLASS OF PARABOLIC EQUATIONS WITH ENERGY SPECIFICATION
}

\author{
ABDELFATAH BOUZIANI \\ Received 3 September 2002
}

This paper deals with weak solution in weighted Sobolev spaces, of three-point boundary value problems which combine Dirichlet and integral conditions, for linear and quasilinear parabolic equations in a domain with curved lateral boundaries. We, firstly, prove the existence, uniqueness, and continuous dependence of the solution for the linear equation. Next, analogous results are established for the quasilinear problem, using an iterative process based on results obtained for the linear problem.

\section{Introduction}

Many physical phenomena are modeled by one-dimensional second-order parabolic equation which involves nonlocal boundary condition of the form

$$
\int_{0}^{b} \theta(x, t) d x=E(t)
$$

the so-called energy specification, where $b \in] 0,1]$ is a constant, $\theta(x, t)$ is an unknown function, and $E(t)$ is a given function.

For heat conduction theory, condition (1.1) represents the internal energy content of a portion of the conductor $[3,9,11,16,17]$. For diffusion processes, the condition is equivalent to the specification of mass in a portion of the domain of diffusion $[3,10,12]$. We note that such problems have other important applications, for instance, in thermoelasticity $[15,18]$, in electrochemistry [14], and in medical science [13].

In this paper, we prove in weighted Sobolev spaces the existence, uniqueness, and continuous dependence of the weak solution of three-point boundary value problem for a class of linear and quasilinear parabolic equations with nonlocal condition in a domain with curved boundaries varying with respect to the time. 
We will first investigate the linear case. Particular cases of it have been treated by several authors; most of the works were directed to strongly generalized solution for two-point boundary value problems $[1,2,3,4,5,6,7,19]$ and to the classical solutions of the heat equation $[8,9,10,11,12,16,17]$. In contrast to previous papers, we consider a weak solution by using a functional analysis method based on a priori estimates. Then, we investigate the quasilinear problem by combining an iterative process with results established for the linear case.

The outline of the paper is as follows. In Section 2, we study the linear problem. In Section 2.1, we give the statement of the problem, the basic assumptions, and some function spaces needed in the remainder of the work. Section 2.2 is devoted to establishing the existence of the solution. The uniqueness and continuous dependence with respect to the data are proved in Section 2.3. In Section 3, analogous investigation for the quasilinear problem is considered.

\section{The linear problem}

2.1. Statement of the problem, hypothesis, and notations. Let $\Gamma_{p}(\tau)$, where $\tau \in I=(0, T)$ and $p=1,2,3$, be nonintersecting curves, varying with time, in the plane $(\xi, \tau)$, such that $\Gamma_{1}(\tau)<\Gamma_{3}(\tau)<\Gamma_{2}(\tau)$. In $Q=\left(\Gamma_{1}(\tau), \Gamma_{2}(\tau)\right) \times I$, we consider the following problem: given a sufficiently smooth data $\Gamma_{p}(p=1,2,3)$, $h, \theta^{0}, \Psi$, and $E$, find the solution $\theta(\xi, \tau)$ of

$$
\begin{gathered}
\frac{\partial \theta}{\partial \tau}-\frac{\partial}{\partial \xi}\left(p_{1}(\xi, \tau) \frac{\partial \theta}{\partial \xi}\right)+p_{2}(\xi, \tau) \frac{\partial \theta}{\partial \xi}+p_{3}(\xi, \tau) \theta=h(\xi, \tau), \quad(\xi, \tau) \in Q \\
\theta(\xi, 0)=\theta^{0}(\xi), \quad \Gamma_{1}(0)<\xi<\Gamma_{2}(0), \\
\theta\left(\Gamma_{2}(\tau), \tau\right)=\Psi(\tau), \quad 0<\tau<T, \\
\int_{\Gamma_{1}(\tau)}^{\Gamma_{3}(\tau)} \theta(\xi, \tau) d \xi=E(\tau), \quad 0<\tau<T,
\end{gathered}
$$

with

$$
\theta^{0}\left(\Gamma_{2}(0)\right)=\Psi(0), \quad \int_{\Gamma_{1}(0)}^{\Gamma_{3}(0)} \theta^{0}(\xi) d \xi=E(0)
$$

Problem (2.1) can be reduced to a problem with homogeneous boundary conditions by setting

$$
\begin{aligned}
\theta(\xi, \tau)= & \Theta(\xi, \tau)+\Psi(\tau) \\
& -\frac{2\left(\Gamma_{2}(\tau)-\xi\right)\left(\Psi(\tau)\left(\Gamma_{3}(\tau)-\Gamma_{1}(\tau)\right)-E(\tau)\right)}{\left(2 \Gamma_{2}(\tau)-\Gamma_{1}(\tau)-\Gamma_{3}(\tau)\right)\left(\Gamma_{3}(\tau)-\Gamma_{1}(\tau)\right)}
\end{aligned}
$$


then the original problem is equivalent to the problem, for $\Theta(\xi, \tau)$ in $\bar{Q}$ :

$$
\begin{gathered}
\frac{\partial \Theta}{\partial \tau}-\frac{\partial}{\partial \xi}\left(p_{1}(\xi, \tau) \frac{\partial \Theta}{\partial \xi}\right)+p_{2}(\xi, \tau) \frac{\partial \Theta}{\partial \xi}+p_{3}(\xi, \tau) \Theta=g(\xi, \tau), \quad(\xi, \tau) \in Q, \\
\Theta(\xi, 0)=\Theta^{0}(\xi), \quad \Gamma_{1}(0)<\xi<\Gamma_{2}(0), \\
\Theta\left(\Gamma_{2}(\tau), \tau\right)=0, \quad 0<\tau<T, \\
\int_{\Gamma_{1}(\tau)}^{\Gamma_{3}(\tau)} \Theta(\xi, \tau) d \xi=0, \quad 0<\tau<T,
\end{gathered}
$$

with

$$
\Theta^{0}\left(\Gamma_{2}(0)\right)=0, \quad \int_{\Gamma_{1}(0)}^{\Gamma_{3}(0)} \Theta^{0}(\xi) d \xi=0 .
$$

We now introduce new variables $x$ and $t$ connected with $\xi$ and $\tau$ by the relations

$$
x= \begin{cases}\frac{b\left(\xi-\Gamma_{1}(\tau)\right)}{\left(\Gamma_{3}(\tau)-\Gamma_{1}(\tau)\right)}, & \Gamma_{1}(\tau)<\xi<\Gamma_{3}(\tau), \\ \frac{(1-b) \xi+b \Gamma_{2}(\tau)-\Gamma_{3}(\tau)}{\left(\Gamma_{2}(\tau)-\Gamma_{3}(\tau)\right)}, & \Gamma_{3}(\tau)<\xi<\Gamma_{2}(\tau),\end{cases}
$$

where $t=\tau$. Under transformation (2.8), the region $Q$ becomes the rectangle $\Omega=\{(x, t): 0<x<1,0<t<T\}$. In the new variable, problem (2.4), (2.5), and (2.6) assumes the form

$$
\begin{gathered}
\frac{\partial u}{\partial t}-\frac{\partial}{\partial x}\left(q_{1}(x, t) \frac{\partial u}{\partial x}\right)+q_{2}(x, t) \frac{\partial u}{\partial x}+q_{3}(x, t) u=f(x, t), \quad(x, t) \in \Omega, \\
u(x, 0)=u^{0}(x), \quad 0<x<1, \\
u(1, t)=0, \quad 0<t<T, \\
\int_{0}^{b} u(x, t) d x=0, \quad 0<t<T,
\end{gathered}
$$

with

$$
u^{0}(1)=0, \quad \int_{0}^{b} u^{0}(x) d x=0
$$

Thus, problem $(2.1)$ for $\theta(\xi, \tau)$ in the region $Q$ has been reduced to problem (2.9), (2.10), and (2.11) for $u(x, t)$ in the rectangle $\Omega$. 
Assumption 2.1. For all $(x, t) \in \bar{\Omega}$, we assume that

$$
0<c_{0} \leq q_{1} \leq c_{1}, \quad\left|\frac{\partial q_{1}}{\partial t}\right| \leq c_{2}, \quad\left|\frac{\partial q_{1}}{\partial x}\right| \leq c_{3}, \quad\left|q_{2}\right| \leq c_{4}, \quad\left|q_{3}\right| \leq c_{5} .
$$

Here and in the rest of the paper $c_{i}$ are positive constants.

We now introduce some function spaces which are related to the study. By $L^{2}(0,1)$ we represent the usual space of Lebesgue square integrable functions on $(0,1)$ whose scalar product and norm will be denoted by $(\cdot, \cdot)_{L^{2}(0,1)}$ and $\|\cdot\|_{L^{2}(0,1)}$, respectively. By $C_{0}(0, b)$ we denote the space of continuous functions with compact support in $(0, b)$. Let $H$ be a Hilbert space with the norm $\|u\|_{H}$ and let $u: I \rightarrow H$ be an abstract function. By $\|u(\cdot, t)\|_{H}$ we denote the norm of the element $u(\cdot, t) \in H$ at a fixed $t$. The space $C(I, H)$ is the set of all continuous functions $u: I \rightarrow H$ with $\|u\|_{C(I, H)}=\max _{t \in[I]}\|u(\cdot, t)\|_{H}<\infty$, and $L^{2}(I, H)$ is the set of the measurable abstract functions $u$ such that $\|u\|_{L^{2}(I, H)}=$ $\left(\int_{I}\|u(\cdot, t)\|_{H}^{2} d t\right)^{1 / 2}<\infty$.

Definition 2.2. Denote by $L_{\rho}^{2}(0,1)$ the weighted $L^{2}$-space with the scalar product

$$
(u, w)_{L_{\rho}^{2}(0,1)}=(\rho u, w)_{L^{2}(0,1)}
$$

and the associated norm

$$
\|u\|_{L_{\rho}^{2}(0,1)}=\|\sqrt{\rho} u\|_{L^{2}(0,1)},
$$

where $\rho(x)$ is a continuous function defined by

$$
\rho(x)= \begin{cases}x^{2}, & 0 \leq x \leq b, \\ b^{2}, & b \leq x \leq 1 .\end{cases}
$$

Definition 2.3. Denote by $H_{\rho}^{1}(0,1)$ the space equipped with the scalar product

$$
(u, w)_{H_{\rho}^{1}(0,1)}:=(u, w)_{L^{2}(0,1)}+\left(\frac{d u}{d x}, \frac{d w}{d x}\right)_{L_{\rho}^{2}(0,1)}
$$

and the associated norm

$$
\|u\|_{H_{\rho}^{1}(0,1)}:=\left(\|u\|_{L^{2}(0,1)}^{2}+\left\|\frac{d u}{d x}\right\|_{L_{\rho}^{2}(0,1)}^{2}\right)^{1 / 2} .
$$

Definition 2.4. Denote by $B_{2}^{1, *}(0, b)$ a completion of the space $C_{0}(0, b)$ for the scalar product

$$
(u, w)_{B_{2}^{1, *}(0, b)}:=\int_{0}^{b} \mathfrak{J}_{x}^{*} u \mathfrak{J}_{x}^{*} w d x
$$


and the associated norm

$$
\|u\|_{B_{2}^{1, *}(0, b)}:=\left\|\mathfrak{I}_{x}^{*} u\right\|_{L^{2}(0, b)}
$$

where $\mathfrak{J}_{x}^{*} u=\int_{x}^{b} u(\eta, \cdot) d \eta$.

As $L^{2}(0,1)$ and $B_{2}^{1, *}(0, b)$ are Hilbert spaces, so are the spaces $L^{2}\left(I, L^{2}(0,1)\right)$ and $L^{2}\left(I, B_{2}^{1, *}(0, b)\right)$.

It is easy to see that

$$
\begin{gathered}
\|u\|_{L_{\rho}^{2}(0,1)} \leq\|u\|_{L^{2}(0,1)}, \\
\|u\|_{B_{2}^{1, *}(0, b)} \leq \frac{b}{\sqrt{2}}\|u\|_{L^{2}(0, b)} \leq \frac{1}{\sqrt{2}}\|u\|_{L^{2}(0, b)}, \\
\|u\|_{B_{2}^{1, *}(0, b)} \leq 2\|x u\|_{L^{2}(0, b)} .
\end{gathered}
$$

2.2. Existence of the solution. First, we make precisely the concept of the solution of problem (2.9), (2.10), and (2.11) we are considering in this paper. For this, we take a function $v(x, t) \in V$, the space of functions belonging to $C^{1}(\bar{\Omega})$, which satisfies the following conditions:

$$
v(x, T)=0, \quad v(1, t)=0, \quad \int_{0}^{b} v(x, t) d x=0 .
$$

It is easy to observe that $\mathfrak{I}_{0}^{*} v=\mathfrak{I}_{b}^{*} v=0$ and $-(d / d x) \mathfrak{I}_{x}^{*} v=v$, for all $t \in \bar{I}$.

We now consider the inner product in $L^{2}\left(I, L^{2}(0,1)\right)$ of (2.9) and the operator

$$
M v= \begin{cases}x^{2} v+2 x \mathfrak{J}_{x}^{*} v, & 0 \leq x \leq b \\ b^{2} v, & b \leq x \leq 1\end{cases}
$$

it follows that

$$
\begin{aligned}
\left(\frac{\partial u}{\partial t}, M v\right)_{L^{2}\left(I, L^{2}(0,1)\right)}-\left(\frac{\partial}{\partial x}\left(q_{1} \frac{\partial u}{\partial x}\right), M v\right)_{L^{2}\left(I, L^{2}(0,1)\right)} & \\
& +\left(q_{2} \frac{\partial u}{\partial x}, M v\right)_{L^{2}\left(I, L^{2}(0,1)\right)}+\left(q_{3} u, M v\right)_{L^{2}\left(I, L^{2}(0,1)\right)} \\
= & (f, M v)_{L^{2}\left(I, L^{2}(0,1)\right)} .
\end{aligned}
$$

We integrate by parts the first two terms on the left-hand side of (2.24). To do this, we assume that $u, v \in C^{1}(\bar{\Omega}), u(x, 0)=0, v(x, T)=0, v(1, t)=0$, and 
578 Parabolic equations with energy specification

$\int_{0}^{b} u(x, t) d x=\int_{0}^{b} v(x, t) d x=0$. In light of the above assumptions, we have

$$
\begin{aligned}
& \left(\frac{\partial u}{\partial t}, M v\right)_{L^{2}\left(I, L^{2}(0,1)\right)}=\left(\frac{\partial u}{\partial t}, v\right)_{L^{2}\left(I, L_{\rho}^{2}(0,1)\right)}+2\left(\frac{\partial u}{\partial t}, x \mathfrak{J}_{x}^{*} v\right)_{L^{2}\left(I, L^{2}(0, b)\right)} \\
& =\left(\frac{\partial u}{\partial t}, v\right)_{L^{2}\left(I, L_{\rho}^{2}(0,1)\right)}+\left.2\left(\mathfrak{J}_{x}^{*} \frac{\partial u}{\partial t}, x \mathfrak{J}_{x}^{*} v\right)_{L^{2}(I)}\right|_{0} ^{b} \\
& +2\left(\mathfrak{J}_{x}^{*} \frac{\partial u}{\partial t}, x v\right)_{L^{2}\left(I, L^{2}(0, b)\right)}-2\left(\frac{\partial u}{\partial t}, v\right)_{L^{2}\left(I, B_{2}^{1, *}(0, b)\right)}, \\
& -\left(\frac{\partial}{\partial x}\left(q_{1} \frac{\partial u}{\partial x}\right), M v\right)_{L^{2}\left(I, L^{2}(0,1)\right)}=-\left.\left(q_{1} \frac{\partial u}{\partial x},\left(x^{2} v+2 x \mathfrak{J}_{x}^{*} v\right)\right)_{L^{2}(I)}\right|_{0} ^{b} \\
& -\left.\left(q_{1} \frac{\partial u}{\partial x}, b^{2} v\right)_{L^{2}(I)}\right|_{b} ^{1} \\
& +\left(q_{1} \frac{\partial u}{\partial x}, \frac{\partial v}{\partial x}\right)_{L^{2}\left(I, L_{\rho}^{2}(0,1)\right)}+\left.2\left(q_{1} u, \mathfrak{J}_{x}^{*} v\right)_{L^{2}(I)}\right|_{0} ^{b} \\
& +2\left(q_{1} u, v\right)_{L^{2}\left(I, L^{2}(0, b)\right)}-2\left(\frac{\partial q_{1}}{\partial x} u, \mathfrak{J}_{x}^{*} v\right)_{L^{2}\left(I, L^{2}(0, b)\right)} .
\end{aligned}
$$

Substituting (2.25) into (2.24), we obtain

$$
\begin{aligned}
\left(\frac{\partial u}{\partial t}, v\right)_{L^{2}\left(I, L_{\rho}^{2}(0,1)\right)}+2\left(\mathfrak{J}_{x}^{*} \frac{\partial u}{\partial t}, x v\right)_{L^{2}\left(I, L^{2}(0, b)\right)}-2\left(\frac{\partial u}{\partial t}, v\right)_{L^{2}\left(I, B_{2}^{1, *}(0, b)\right)} \\
+\left(q_{1} \frac{\partial u}{\partial x}, \frac{\partial v}{\partial x}\right)_{L^{2}\left(I, L_{\rho}^{2}(0,1)\right)}+2\left(q_{1} u, v\right)_{L^{2}\left(I, L^{2}(0, b)\right)} \\
\quad-2\left(\frac{\partial q_{1}}{\partial x} u, \mathfrak{J}_{x}^{*} v\right)_{L^{2}\left(I, L^{2}(0, b)\right)}+\left(q_{2} \frac{\partial u}{\partial x}, v\right)_{L^{2}\left(I, L_{\rho}^{2}(0,1)\right)} \\
+2\left(q_{2} \frac{\partial u}{\partial x}, x \mathfrak{J}_{x}^{*} v\right)_{L^{2}\left(I, L^{2}(0, b)\right)}+\left(q_{3} u, v\right)_{L^{2}\left(I, L_{\rho}^{2}(0,1)\right)} \\
\quad+2\left(q_{3} u, x \mathfrak{J}_{x}^{*} v\right)_{L^{2}\left(I, L^{2}(0, b)\right)} \\
=(f, v)_{L^{2}\left(I, L_{\rho}^{2}(0,1)\right)}+2\left(f, x \mathfrak{J}_{x}^{*} v\right)_{L^{2}\left(I, L^{2}(0, b)\right)}+\left(u^{0}, M v(\cdot, 0)\right)_{L^{2}(0,1)}
\end{aligned}
$$

This equality may be written as

$$
A(u, v)=(f, v)_{L^{2}\left(I, L_{\rho}^{2}(0,1)\right)}+2\left(f, x \mathfrak{I}_{x}^{*} v\right)_{L^{2}\left(I, L^{2}(0, b)\right)}+\left(u^{0}, M v(\cdot, 0)\right)_{L^{2}(0,1)},
$$

where $A(u, v)$ is the left-hand side of (2.26).

Definition 2.5. We say that $u \in L^{2}\left(I, H_{\rho}^{1}(0,1)\right)$ is a weak solution of problem (2.9), (2.10), and (2.11) if identity (2.27) holds for all $v \in V$, and $u$ verifies conditions (2.10). 
We now give an approximation of problem (2.9), (2.10), and (2.11). To this end, we suppose that there are sequences of functions $f_{n} \in C(\bar{\Omega})$ and $u_{n}^{0} \in C^{1}[0,1]$, $n=1,2, \ldots$, such that

$$
\begin{gathered}
f_{n} \longrightarrow f \quad \text { in } L_{\rho}^{2}(0,1), \forall t \in \bar{I}, \\
u_{n}^{0} \longrightarrow u^{0} \quad \text { in } H_{\rho}^{1}(0,1), \\
\frac{\partial u_{n}}{\partial t}-\frac{\partial}{\partial x}\left(q_{1} \frac{\partial u_{n}}{\partial x}\right)+q_{2} \frac{\partial u_{n}}{\partial x}+q_{3} u_{n}=f_{n}(x, t), \\
u_{n}(x, 0)=u_{n}^{0}(x), \quad u_{n}(1, t)=0, \quad \int_{0}^{b} u_{n}(x, t) d x=0 .
\end{gathered}
$$

Problem (2.29) possesses a unique classical solution $u_{n}=u_{n}(x, t)$. For the proof, we refer the reader to [17].

Taking the difference of (2.29) for $n=i$ and $n=j$, we get

$$
\begin{gathered}
\frac{\partial u_{i j}}{\partial t}-\frac{\partial}{\partial x}\left(q_{1} \frac{\partial u_{i j}}{\partial x}\right)+q_{2} \frac{\partial u_{i j}}{\partial x}+q_{3} u_{i j}=f_{i j}(x, t), \\
u_{i j}(x, 0)=u_{i j}^{0}(x), \quad u_{i j}(1, t)=0, \quad \int_{0}^{b} u_{i j}(x, t) d x=0,
\end{gathered}
$$

with $u_{i j}(x, t)=u_{i}(x, t)-u_{j}(x, t), f_{i j}(x, t)=f_{i}(x, t)-f_{j}(x, t)$, and $u_{i j}^{0}(x)=u_{i}^{0}(x)-$ $u_{j}^{0}(x)$.

We must derive some a priori estimates for $u_{i j}$. To this end, we first compute the integral $\int_{0}^{t}\left(f(\cdot, \tau), M\left(\partial u_{i j}(\cdot, \tau) / \partial t\right)\right)_{L^{2}(0,1)} d \tau$ :

$$
\begin{aligned}
& \int_{0}^{t}\left(\frac{\partial u_{i j}(\cdot, \tau)}{\partial t}, M \frac{\partial u_{i j}(\cdot, \tau)}{\partial t}\right)_{L^{2}(0,1)} d \tau \int_{0}^{t}\left(\left\|\frac{\partial u_{i j}(\cdot, \tau)}{\partial t}\right\|_{L_{\rho}^{2}(0,1)}^{2}+\left\|\frac{\partial u_{i j}(\cdot, \tau)}{\partial t}\right\|_{B_{2}^{1, *}(0, b)}^{2}\right) d \tau \\
& \int_{0}^{t}\left(\frac{\partial}{\partial x}\left(q_{1} \frac{\partial u_{i j}(\cdot, \tau)}{\partial x}\right), M \frac{\partial u_{i j}(\cdot, \tau)}{\partial t}\right)_{L^{2}(0,1)} d \tau \\
&=\int_{0}^{b} q_{1} u_{i j}^{2}(x, t) d x \\
& \quad+\frac{1}{2} \int_{0}^{1} q_{1}(x, t) \rho(x)\left(\frac{\partial u_{i j}(x, t)}{\partial t}\right)^{2} d x-\int_{0}^{b} q_{1}(x, 0)\left(u_{i j}^{0}(x)\right)^{2} d x \\
& \quad-\frac{1}{2} \int_{0}^{1} q_{1}(x, 0) \rho(x)\left(\frac{d u_{i j}^{0}(x)}{\partial x}\right)^{2} d x-\int_{0}^{t} \int_{0}^{b} \frac{\partial q_{1}}{\partial t} u_{i j}^{2} d x d \tau \\
& \quad-\frac{1}{2} \int_{0}^{t} \int_{0}^{b} \frac{\partial q_{1}}{\partial t} \rho(x)\left(\frac{\partial u_{i j}}{\partial x}\right)^{2} d x d \tau \\
& \quad-2 \int_{0}^{t}\left(\frac{\partial q_{1}}{\partial x} u_{i j}(\cdot, \tau), \mathfrak{J}_{x}^{*} \frac{\partial u_{i j}(\cdot, \tau)}{\partial t}\right)_{L^{2}(0, b)} d \tau .
\end{aligned}
$$


From identities (2.31), we get

$$
\begin{aligned}
& \int_{0}^{t}\left(\left\|\frac{\partial u_{i j}(\cdot, \tau)}{\partial t}\right\|_{L_{\rho}^{2}(0,1)}^{2}+\left\|\frac{\partial u_{i j}(\cdot, \tau)}{\partial t}\right\|_{B_{2}^{1, *}(0, b)}^{2}\right) d \tau \\
&+\frac{1}{2} \int_{0}^{1} q_{1}(x, t) \rho(x)\left(\frac{\partial u_{i j}(x, t)}{\partial t}\right)^{2} d x+\int_{0}^{b} q_{1} u_{i j}^{2} d x \\
&=\int_{0}^{t}\left(f(\cdot, \tau), M \frac{\partial u_{i j}(\cdot, \tau)}{\partial t}\right)_{L^{2}(0,1)} d \tau+\int_{0}^{b} q_{1}(x, 0)\left(u_{i j}^{0}(x)\right)^{2} d x \\
&+\frac{1}{2} \int_{0}^{1} q_{1}(x, 0) \rho(x)\left(\frac{d u_{i j}^{0}(x)}{d x}\right)^{2} d x+\int_{0}^{t} \int_{0}^{b} \frac{\partial q_{1}}{\partial t} u_{i j}^{2} d x d \tau \\
&+\frac{1}{2} \int_{0}^{t} \int_{0}^{b} \frac{\partial q_{1}}{\partial t} \rho(x)\left(\frac{\partial u_{i j}}{\partial x}\right)^{2} d x d \tau \\
&+2 \int_{0}^{t}\left(\frac{\partial q_{1}}{\partial x} u_{i j}(\cdot, \tau), \mathfrak{J}_{x}^{*} \frac{\partial u_{i j}(\cdot, \tau)}{\partial t}\right)_{L^{2}(0, b)}^{d \tau}\left(u_{i j}(\cdot, \tau)\right. \\
&+ 2 \int_{0}^{t}\left(\left(q_{2} \frac{\partial u_{i j}(\cdot, \tau)}{\partial x}+q_{3} u_{i j}(\cdot, \tau)\right), \frac{\partial t}{\partial t} d \tau\right. \\
&+2 \int_{0}^{t}\left(x\left(q_{2} \frac{\partial u_{i j}(\cdot, \tau)}{\partial x}+q_{3} u_{i j}(\cdot, \tau)\right), \mathfrak{J}_{x}^{*} \frac{\partial u_{i j}(\cdot, \tau)}{\partial t}\right)_{L^{2}(0, b)} d \tau
\end{aligned}
$$

According to the Cauchy inequality, the first and the last three integrals on the right-hand side of (2.32) are controlled from above as follows:

$$
\begin{aligned}
& \int_{0}^{t}\left(f(\cdot, \tau), M \frac{\partial u_{i j}(\cdot, \tau)}{\partial t}\right)_{L^{2}(0,1)} d \tau \\
& \leq\left(\frac{\varepsilon_{1}}{2}+\varepsilon_{2}\right) \int_{0}^{t}\left\|f_{i j}(\cdot, \tau)\right\|_{L_{\rho}^{2}(0,1)}^{2} d \tau+\frac{1}{2 \varepsilon_{1}} \int_{0}^{t}\left\|\frac{\partial u_{i j}(\cdot, \tau)}{\partial t}\right\|_{L_{\rho}^{2}(0,1)}^{2} d \tau \\
& \quad+\frac{1}{\varepsilon_{2}} \int_{0}^{t}\left\|\frac{\partial u_{i j}(\cdot, \tau)}{\partial t}\right\|_{B_{2}^{1, *}(0, b)}^{2} d \tau \\
& 2 \int_{0}^{t}\left(\frac{\partial q_{1}}{\partial x} u_{i j}(\cdot, \tau), \mathfrak{J}_{x}^{*} \frac{\partial u_{i j}(\cdot, \tau)}{\partial t}\right)_{L^{2}(0, b)} d \tau \\
& \leq \varepsilon_{3} \int_{0}^{t} \int_{0}^{b}\left(\frac{\partial q_{1}}{\partial x}\right)^{2} u_{i j}^{2} d x d \tau+\frac{1}{\varepsilon_{3}} \int_{0}^{t}\left\|\frac{\partial u_{i j}(\cdot, \tau)}{\partial t}\right\|_{B_{2}^{1, *}(0, b)}^{2} d \tau, \\
& 2 \int_{0}^{t}\left(\left(q_{2} \frac{\partial u_{i j}(\cdot, \tau)}{\partial x}+q_{3} u_{i j}(\cdot, \tau)\right), \frac{\partial u_{i j}(\cdot, \tau)}{\partial t}\right)_{L_{\rho}^{2}(0,1)} d \tau \\
& \leq 2 \varepsilon_{4} \int_{0}^{t} \int_{0}^{1}\left(q_{2}^{2} \rho\left(\frac{\partial u_{i j}}{\partial x}\right)^{2}+q_{3}^{2} u_{i j}^{2}\right) d x d \tau+\frac{1}{\varepsilon_{4}} \int_{0}^{t}\left\|\frac{\partial u_{i j}(\cdot, \tau)}{\partial t}\right\|_{L_{\rho}^{2}(0,1)}^{2} d \tau,
\end{aligned}
$$




$$
\begin{aligned}
& 2 \int_{0}^{t}\left(x\left(q_{2} \frac{\partial u_{i j}(\cdot, \tau)}{\partial x}+q_{3} u_{i j}(\cdot, \tau)\right), \mathfrak{J}_{x}^{*} \frac{\partial u_{i j}(\cdot, \tau)}{\partial t}\right)_{L^{2}(0, b)} d \tau \\
& \quad \leq 2 \varepsilon_{5} \int_{0}^{t} \int_{0}^{1}\left(q_{2}^{2} \rho(x)\left(\frac{\partial u_{i j}}{\partial x}\right)^{2}+q_{3}^{2} u_{i j}^{2}\right) d x d \tau+\frac{1}{\varepsilon_{5}} \int_{0}^{t}\left\|\frac{\partial u_{i j}(\cdot, \tau)}{\partial t}\right\|_{B_{2}^{1, *}(0, b)}^{2} d \tau
\end{aligned}
$$

Substituting (2.33) into (2.32) and taking into account Assumption 2.1, we get by choosing $\varepsilon_{1}=2, \varepsilon_{2}=\varepsilon_{3}=3, \varepsilon_{4}=4$, and $\varepsilon_{5}=3$

$$
\begin{gathered}
\frac{1}{2} \int_{0}^{t}\left\|\frac{\partial u_{i j}(\cdot, \tau)}{\partial t}\right\|_{L_{\rho}^{2}(0,1)}^{2} d \tau+c_{0}\left\|u_{i j}(\cdot, t)\right\|_{L_{\rho}^{2}(0, b)}^{2}+\frac{c_{0}}{2}\left\|\frac{\partial u_{i j}(\cdot, t)}{\partial x}\right\|_{L_{\rho}^{2}(0,1)}^{2} \\
\leq 4 \int_{0}^{t}\left\|f_{i j}(\cdot, \tau)\right\|_{L_{\rho}^{2}(0,1)}^{2} d \tau+c_{1}\left\|u_{i j}^{0}\right\|_{L^{2}(0, b)}^{2}+\frac{c_{1}}{2}\left\|\frac{d u_{i j}^{0}}{d x}\right\|_{L_{\rho}^{2}(0,1)}^{2} \\
+\left(c_{2}+3 c_{3}^{2}+14 c_{5}^{2}\right) \int_{0}^{t}\left\|u_{i j}(\cdot, \tau)\right\|_{L^{2}(0, b)}^{2} d \tau \\
+\left(\frac{c_{2}}{2}+14 c_{4}^{2}\right) \int_{0}^{t}\left\|\frac{\partial u_{i j}(\cdot, \tau)}{\partial x}\right\|_{L_{\rho}^{2}(0,1)}^{2} d \tau .
\end{gathered}
$$

In light of the elementary inequality

$$
\begin{aligned}
& \frac{b^{2}}{4}\left\|u_{i j}(\cdot, t)\right\|_{L^{2}(b, 1)}^{2} \\
& \quad \leq \frac{b^{2}}{4}\left\|u_{i j}^{0}\right\|_{L^{2}(b, 1)}^{2}+\frac{b^{2}}{4} \int_{0}^{t}\left\|u_{i j}(\cdot, \tau)\right\|_{L^{2}(b, 1)}^{2} d \tau+\frac{b^{2}}{4} \int_{0}^{t}\left\|\frac{\partial u_{i j}(\cdot, \tau)}{\partial t}\right\|_{L^{2}(b, 1)}^{2} d t \\
& \quad \leq \frac{b^{2}}{4}\left\|u_{i j}^{0}\right\|_{L^{2}(b, 1)}^{2}+\frac{b^{2}}{4} \int_{0}^{t}\left\|u_{i j}(\cdot, \tau)\right\|_{L^{2}(b, 1)}^{2} d \tau+\frac{1}{4} \int_{0}^{t}\left\|\frac{\partial u_{i j}(\cdot, \tau)}{\partial t}\right\|_{L_{\rho}^{2}(0,1)}^{2} d t
\end{aligned}
$$

the last estimate becomes

$$
\begin{aligned}
& \int_{0}^{t}\left\|\frac{\partial u_{i j}(\cdot, \tau)}{\partial t}\right\|_{L_{\rho}^{2}(0,1)}^{2} d \tau+\left\|u_{i j}(\cdot, t)\right\|_{H_{\rho}^{1}(0,1)}^{2} \\
& \quad \leq c_{6}\left(\int_{0}^{t}\left\|f_{i j}(\cdot, \tau)\right\|_{L_{\rho}^{2}(0,1)}^{2} d \tau+\left\|u_{i j}^{0}\right\|_{H_{\rho}^{1}(0,1)}^{2}\right)+c_{7} \int_{0}^{t}\left\|u_{i j}(\cdot, \tau)\right\|_{H_{\rho}^{1}(0,1)}^{2} d \tau,
\end{aligned}
$$

where

$$
\begin{aligned}
c_{6} & =\frac{\max \left(4, c_{1}, b^{2} / 4\right)}{\min \left(c_{0} / 2, b^{2} / 4\right)} \\
c_{7} & =\frac{\max \left(c_{2}+3 c_{3}^{2}+14 c_{5}^{2}, b^{2} / 4, c_{2} / 2+14 c_{4}^{2}\right)}{\min \left(c_{0} / 2, b^{2} / 4\right)} .
\end{aligned}
$$


582 Parabolic equations with energy specification

Therefore, from Gronwall's lemma, we come to the conclusion that

$$
\begin{aligned}
& \int_{0}^{t}\left\|\frac{\partial u_{i j}(\cdot, \tau)}{\partial t}\right\|_{L_{\rho}^{2}(0,1)}^{2} d \tau+\left\|u_{i j}(\cdot, t)\right\|_{H_{\rho}^{1}(0,1)}^{2} \\
& \quad \leq c_{6} \exp \left(c_{7} T\right)\left(\left\|f_{i j}\right\|_{L^{2}\left(I, L_{\rho}^{2}(0,1)\right)}^{2}+\left\|u_{i j}^{0}\right\|_{H_{\rho}^{1}(0,1)}^{2}\right) .
\end{aligned}
$$

If we omit the first term on the left-hand side of (2.38) and integrate the result over $I$, it yields

$$
\left\|u_{i j}\right\|_{L^{2}\left(I, H_{\rho}^{1}(0,1)\right)}^{2} \leq c_{6} T \exp \left(c_{7} T\right)\left(\left\|f_{i j}\right\|_{L^{2}\left(I, L_{\rho}^{2}(0,1)\right)}^{2}+\left\|u_{i j}^{0}\right\|_{H_{\rho}^{1}(0,1)}^{2}\right) .
$$

On the other hand, if in the left-hand side of (2.38) we take the upper bound with respect to $t$, from 0 to $T$, since the right-hand side of the inequality does not depend on $t$, we obtain

$$
\begin{aligned}
& \left\|\frac{\partial u_{i j}}{\partial t}\right\|_{L^{2}\left(I, L_{\rho}^{2}(0,1)\right)}^{2}+\left\|u_{i j}\right\|_{C\left(I, H_{\rho}^{1}(0,1)\right)}^{2} \\
& \quad \leq c_{6} \exp \left(c_{7} T\right)\left(\left\|f_{i j}\right\|_{L^{2}\left(I, L_{\rho}^{2}(0,1)\right)}^{2}+\left\|u_{i j}^{0}\right\|_{H_{\rho}^{1}(0,1)}^{2}\right) .
\end{aligned}
$$

Thus we have proved the following theorem.

Theorem 2.6. Suppose that Assumption 2.1 holds. Then, the solution of problem (2.30) satisfies the following estimates, for $T>0$ :

$$
\begin{aligned}
\left\|u_{i j}\right\|_{L^{2}\left(I, H_{\rho}^{1}(0,1)\right)}^{2} & \leq c_{8}\left(\left\|f_{i j}\right\|_{L^{2}\left(I, L_{\rho}^{2}(0,1)\right)}^{2}+\left\|u_{i j}^{0}\right\|_{H_{\rho}^{1}(0,1)}^{2}\right), \\
\left\|u_{i j}\right\|_{C\left(I, H_{\rho}^{1}(0,1)\right)}^{2} & \leq c_{9}\left(\left\|f_{i j}\right\|_{L^{2}\left(I, L_{\rho}^{2}(0,1)\right)}^{2}+\left\|u_{i j}^{0}\right\|_{H_{\rho}^{1}(0,1)}^{2}\right), \\
\left\|\frac{\partial u_{i j}}{\partial t}\right\|_{L^{2}\left(I, L_{\rho}^{2}(0,1)\right)}^{2} & \leq c_{9}\left(\left\|f_{i j}\right\|_{L^{2}\left(I, L_{\rho}^{2}(0,1)\right)}^{2}+\left\|u_{i j}^{0}\right\|_{H_{\rho}^{1}(0,1)}^{2}\right),
\end{aligned}
$$

where $c_{8}=c_{6} T \exp \left(c_{7} T\right)$ and $c_{9}=c_{6} \exp \left(c_{7} T\right)$.

From estimates (2.41), it follows immediately that $\left\{u_{n}\right\}_{n}$ is a Cauchy sequence. Thus we have the following corollary.

COROLlary 2.7. Under the assumptions of Theorem 2.6, the sequence $\left\{u_{n}\right\}_{n}$ converges to $\tilde{u}$ in the following sense:

$$
\begin{array}{cc}
u_{n} \longrightarrow \tilde{u} & \text { in } L^{2}\left(I, H_{\rho}^{1}(0,1)\right), \\
u_{n} \longrightarrow \tilde{u} & \text { in } C\left(I, H_{\rho}^{1}(0,1)\right), \\
\frac{\partial u_{n}}{\partial t} \longrightarrow \frac{\partial \tilde{u}}{\partial t} & \text { in } L^{2}\left(I, L_{\rho}^{2}(0,1)\right) .
\end{array}
$$

We must prove that the limit function $\tilde{u}(x, t)$ is a solution of problem (2.9), (2.10), and (2.11) in the sense of Definition 2.5. For this purpose, we consider 
the weak formulation of problem (2.29)

$$
A\left(u_{n}, v\right)=\left(f_{n}, v\right)_{L^{2}\left(I, L_{\rho}^{2}(0,1)\right)}+2\left(f_{n}, x \mathfrak{J}_{x}^{*} v\right)_{L^{2}\left(I, L^{2}(0, b)\right)}+\left(u_{n}^{0}, M v(\cdot, 0)\right)_{L^{2}(0,1)} .
$$

However, $u_{n}=\left(u_{n}-\tilde{u}\right)+\tilde{u}, u_{n}^{0}=\left(u_{n}^{0}-u^{0}\right)+u^{0}$, and $f_{n}=\left(f_{n}-f\right)+f$, then it follows from the last identity that

$$
\begin{aligned}
A\left(u_{n}-\tilde{u}, v\right)+A(\tilde{u}, v)= & \left(f_{n}-f, v\right)_{L^{2}\left(I, L_{\rho}^{2}(0,1)\right)}+(f, v)_{L^{2}\left(I, L_{\rho}^{2}(0,1)\right)} \\
& +2\left(f_{n}-f, x \mathfrak{J}_{x}^{*} v\right)_{L^{2}\left(I, L^{2}(0, b)\right)}+2\left(f, x \mathfrak{I}_{x}^{*} v\right)_{L^{2}\left(I, L^{2}(0, b)\right)} \\
& +\left(u_{n}^{0}-u^{0}, M v(\cdot, 0)\right)_{L^{2}(0,1)}+\left(u^{0}, M v(\cdot, 0)\right)_{L^{2}(0,1)}
\end{aligned}
$$

In light of the Schwarz inequality and inequalities (2.21), we get the following estimates:

$$
\begin{aligned}
& A\left(u_{n}-\tilde{u}, v\right)=\left(\frac{\partial\left(u_{n}-\tilde{u}\right)}{\partial t}, v\right)_{L^{2}\left(I, L_{\rho}^{2}(0,1)\right)}+2\left(\mathfrak{J}_{x}^{*} \frac{\partial\left(u_{n}-\tilde{u}\right)}{\partial t}, x v\right)_{L^{2}\left(I, L^{2}(0, b)\right)} \\
& -2\left(\frac{\partial\left(u_{n}-\tilde{u}\right)}{\partial t}, v\right)_{L^{2}\left(I, B_{2}^{1, *}(0, b)\right)}+\left(q_{1} \frac{\partial\left(u_{n}-\tilde{u}\right)}{\partial x}, \frac{\partial v}{\partial x}\right)_{L^{2}\left(I, L_{\rho}^{2}(0,1)\right)} \\
& +\left(q_{2} \frac{\partial\left(u_{n}-\tilde{u}\right)}{\partial x}, v\right)_{L^{2}\left(I, L_{\rho}^{2}(0,1)\right)}+2\left(q_{2} \frac{\partial\left(u_{n}-\tilde{u}\right)}{\partial x}, x \mathfrak{I}_{x}^{*} v\right)_{L^{2}\left(I, L^{2}(0, b)\right)} \\
& +2\left(q_{1}\left(u_{n}-\tilde{u}\right), v\right)_{L^{2}\left(I, L^{2}(0, b)\right)}-2\left(\frac{\partial q_{1}}{\partial x}\left(u_{n}-\tilde{u}\right), \mathfrak{J}_{x}^{*} v\right)_{L^{2}\left(I, L^{2}(0, b)\right)} \\
& +\left(q_{3}\left(u_{n}-\tilde{u}\right), v\right)_{L^{2}\left(I, L_{\rho}^{2}(0,1)\right)}+2\left(q_{3}\left(u_{n}-\tilde{u}\right), x \mathfrak{J}_{x}^{*} v\right)_{L^{2}\left(I, L^{2}(0, b)\right)} \\
& \leq \max \left(5+\sqrt{2}, c_{1}+(1+\sqrt{2}) c_{4}, 2 c_{1}+\sqrt{2} c_{3}+(1+\sqrt{2}) c_{5}\right) \\
& \times\left(\left\|\frac{\partial\left(u_{n}-\tilde{u}\right)}{\partial t}\right\|_{L^{2}\left(I, L_{\rho}^{2}(0,1)\right)}+\left\|\tilde{u}-u_{n}\right\|_{L^{2}\left(I, H_{\rho}^{1}(0,1)\right)}\right)\|v\|_{L^{2}\left(I, H_{\rho}^{1}(0,1)\right)}, \\
& \left(u_{n}^{0}-u^{0}, M v(\cdot, 0)\right)_{L^{2}(0,1)} \\
& \leq(1+\sqrt{2})\left\|u_{n}^{0}-u^{0}\right\|_{H_{\rho}^{1}(0,1)} \cdot\|v(\cdot, 0)\|_{L^{2}(0,1)}, \\
& \left(f_{n}-f, v\right)_{L^{2}\left(I, L_{\rho}^{2}(0,1)\right)}+2\left(f_{n}-f, x \mathfrak{J}_{x}^{*} v\right)_{L^{2}\left(I, L^{2}(0, b)\right)} \\
& \leq(1+\sqrt{2})\left\|f_{n}-f\right\|_{L^{2}\left(I, L_{\rho}^{2}(0,1)\right)}\|v\|_{L^{2}\left(I, L_{\rho}^{2}(0,1)\right)} .
\end{aligned}
$$

Therefore, if we pass to the limit in equality (2.46), by taking into account the limit relations (2.42) and (2.44), and estimates (2.47), we conclude that $\tilde{u}$ satisfies equality (2.27). On the other hand, it follows from Corollary 2.7 that $\int_{0}^{t} \tilde{u}(x, t) d x$ is in $C(\bar{\Omega})$; from which we deduce that $\tilde{u}(1, t)=0$ almost everywhere. 
It remains to prove that $\tilde{u}$ satisfies the initial condition. Let

$$
\begin{aligned}
\left\|\tilde{u}(\cdot, 0)-u^{0}\right\|_{H_{\rho}^{1}(0,1)} & \leq\left\|\tilde{u}(\cdot, 0)-u_{n}(\cdot, 0)\right\|_{H_{\rho}^{1}(0,1)}+\left\|u_{n}^{0}-u^{0}\right\|_{H_{\rho}^{1}(0,1)} \\
& \leq\left\|\tilde{u}-u_{n}\right\|_{C\left(I, H_{\rho}^{1}(0,1)\right)}+\left\|u_{n}^{0}-u^{0}\right\|_{H_{\rho}^{1}(0,1)} .
\end{aligned}
$$

Passing to the limit as $n \rightarrow+\infty$ in the above inequality, by taking into account (2.43) and (2.28b), we get $\tilde{u}(x, 0)=u^{0}(x)$.

We have thus proved the following theorem.

Theorem 2.8. If $u^{0}$ belongs to $H_{\rho}^{1}(0,1)$ and $f$ to $L^{2}\left(I, L_{\rho}^{2}(0,1)\right)$, then there exists a weak solution $u$ of problem (2.9), (2.10), and (2.11) possessing the following properties:

$$
\begin{aligned}
A(u, v)= & (f, v)_{L^{2}\left(I, L_{\rho}^{2}(0,1)\right)}+2\left(f, x \mathfrak{J}_{x}^{*} v\right)_{L^{2}\left(I, L^{2}(0, b)\right)} \\
& +\left(u^{0}, M v(\cdot, 0)\right)_{L^{2}(0,1)}, \quad u \in L^{2}\left(I, H_{\rho}^{1}(0,1)\right) .
\end{aligned}
$$

Moreover,

$$
u \in C\left(I, H_{\rho}^{1}(0,1)\right), \quad \frac{\partial u}{\partial t} \in L^{2}\left(I, L_{\rho}^{2}(0,1)\right)
$$

2.3. Continuous dependence and uniqueness. We first establish the continuous dependence of the solution with respect to the data. The uniqueness then follows directly.

Theorem 2.9. Suppose that Assumption 2.1 holds. Let $\left(f, u_{0}\right),\left(f^{*}, u_{0}^{*}\right) \in L^{2}(I$, $\left.H_{\rho}^{1}(0,1)\right) \times H_{\rho}^{1}(0,1)$ and let $u, u^{*}$ be two solutions of problem (2.9), (2.10), and (2.11) corresponding, respectively, to the above data. Then

$$
\begin{aligned}
&\left\|u-u^{*}\right\|_{L^{2}\left(I, H_{\rho}^{1}(0,1)\right)}^{2} \leq c_{8}\left(\left\|f-f^{*}\right\|_{L^{2}\left(I, L_{\rho}^{2}(0,1)\right)}^{2}+\left\|u^{0}-u^{* 0}\right\|_{H_{\rho}^{1}(0,1)}^{2}\right), \\
&\left\|u-u^{*}\right\|_{C\left(I, H_{\rho}^{1}(0,1)\right)}^{2} \leq c_{9}\left(\left\|f-f^{*}\right\|_{L^{2}\left(I, L_{\rho}^{2}(0,1)\right)}^{2}+\left\|u^{0}-u^{* 0}\right\|_{H_{\rho}^{1}(0,1)}^{2}\right), \\
&\left\|\frac{\partial u}{\partial t}-\frac{\partial u^{*}}{\partial t}\right\|_{L^{2}\left(I, L_{\rho}^{2}(0,1)\right)}^{2} \leq c_{9}\left(\left\|f-f^{*}\right\|_{L^{2}\left(I, L_{\rho}^{2}(0,1)\right)}^{2}+\left\|u^{0}-u^{* 0}\right\|_{H_{\rho}^{1}(0,1)}^{2}\right) .
\end{aligned}
$$

Proof. Let $\left\{u_{n}(x, t)\right\}_{n}$ be a sequence of classical solutions of problem (2.29), converging to the weak solution. Therefore, we have the analogue of estimates (2.41), with $u_{i j}$ replaced by $u_{n}$. If we pass to the limit, in the resulting inequalities, as $n \rightarrow \infty$, by taking into account (2.28), we obtain

$$
\begin{aligned}
\|u\|_{L^{2}\left(I, H_{\rho}^{1}(0,1)\right)}^{2} & \leq c_{8}\left(\|f\|_{L^{2}\left(I, L_{\rho}^{2}(0,1)\right)}^{2}+\left\|u^{0}\right\|_{H_{\rho}^{1}(0,1)}^{2}\right), \\
\|u\|_{C\left(I, H_{\rho}^{1}(0,1)\right)}^{2} & \leq c_{9}\left(\|f\|_{L^{2}\left(I, L_{\rho}^{2}(0,1)\right)}^{2}+\left\|u^{0}\right\|_{H_{\rho}^{1}(0,1)}^{2}\right), \\
\left\|\frac{\partial u}{\partial t}\right\|_{L^{2}\left(I, L_{\rho}^{2}(0,1)\right)}^{2} & \leq c_{9}\left(\|f\|_{L^{2}\left(I, L_{\rho}^{2}(0,1)\right)}^{2}+\left\|u^{0}\right\|_{H_{\rho}^{1}(0,1)}^{2}\right) .
\end{aligned}
$$


Set $\hat{u}=u-u^{*}, \hat{u}^{0}=u^{0}-u^{0 *}$, and $\hat{f}=f-f^{*}$. From (2.52), with $u$ replaced by $\hat{u}, u^{0}$ by $\hat{u}^{0}$, and $f$ by $\hat{f}$, we get inequalities (2.51).

COROLlary 2.10. Under the assumptions of Theorem 2.8, the weak solution of problem (2.9), (2.10), and (2.11) is unique.

\section{The quasilinear problem}

3.1. Statement of the problem. In this section, we consider a boundary value problem for quasilinear parabolic equation which combines Dirichlet and integral conditions in a domain with curved boundaries. Precisely, we state the problem as follows. Find a function $\theta$ satisfying

$$
\begin{gathered}
\frac{\partial \theta}{\partial \tau}-\frac{\partial}{\partial \xi}\left(p_{1}(\xi, \tau) \frac{\partial \theta}{\partial \xi}\right)+p_{2}(\xi, \tau) \frac{\partial \theta}{\partial \xi}+p_{3}(\xi, \tau) \theta=h\left(\xi, \tau, \theta, \frac{\partial \theta}{\partial \xi}\right), \quad(\xi, \tau) \in Q, \\
\theta(\xi, 0)=\theta^{0}(\xi), \quad \Gamma_{1}(0)<\xi<\Gamma_{2}(0), \\
\theta\left(\Gamma_{2}(\tau), \tau\right)=\Psi(\tau), \quad 0<\tau<T, \\
\int_{\Gamma_{1}(\tau)}^{\Gamma_{3}(\tau)} \theta(\xi, t) d \xi=E(t), \quad 0<\tau<T .
\end{gathered}
$$

Here, we conserve the notations given in Section 2.

As in Section 2.1, we reduce problem (3.1) to the following:

$$
\begin{gathered}
\frac{\partial u}{\partial t}-\frac{\partial}{\partial x}\left(q_{1}(x, t) \frac{\partial u}{\partial x}\right)+q_{2}(x, t) \frac{\partial u}{\partial x}+q_{3}(x, t) u=f\left(x, t, u, \frac{\partial u}{\partial x}\right), \quad(x, t) \in \Omega, \\
u(x, 0)=u^{0}(x), \quad 0<x<1, \\
u(1, t)=0, \quad 0<t<T, \\
\int_{0}^{b} u(x, t) d x=0, \quad 0<t<T .
\end{gathered}
$$

Consider now the following auxiliary problem:

$$
\begin{gathered}
\frac{\partial \zeta}{\partial t}-\frac{\partial}{\partial x}\left(q_{1}(x, t) \frac{\partial \zeta}{\partial x}\right)+q_{2}(x, t) \frac{\partial \zeta}{\partial x}+q_{3}(x, t) \zeta=0 \\
\zeta(x, 0)=u^{0}(x), \quad 0<x<1 \\
\zeta(1, t)=0, \quad 0<t<T \\
\int_{0}^{b} \zeta(x, t) d x=0, \quad 0<t<T .
\end{gathered}
$$


Let $\omega=u-\zeta$, where $u$ is the solution of problem (3.2). Then it follows from (3.2) and (3.3) that $\omega$ verifies

$$
\begin{gathered}
\frac{\partial \omega}{\partial t}-\frac{\partial}{\partial x}\left(q_{1}(x, t) \frac{\partial \omega}{\partial x}\right)+q_{2}(x, t) \frac{\partial \omega}{\partial x}+q_{3}(x, t) \omega=f\left(x, t, \omega+\zeta, \frac{\partial \omega}{\partial x}+\frac{\partial \zeta}{\partial x}\right) \\
\omega(x, 0)=0, \quad \omega(1, t)=0, \quad \int_{0}^{b} \omega(x, t) d x=0 .
\end{gathered}
$$

Section 2 implies that problem (3.3) admits a unique weak solution that depends continuously with respect to the initial condition. Thus it remains to establish the proof for problem (3.4). For this purpose, we employ the following iteration procedure.

Let $\omega^{(0)}=0$ and let $\left\{\omega^{(n)}\right\}_{n}$ be defined as follows: if $\omega^{(n-1)}$ is given, then solve the following problem:

$$
\begin{gathered}
\frac{\partial \omega^{(n)}}{\partial t}-\frac{\partial}{\partial x}\left(q_{1} \frac{\partial \omega^{(n)}}{\partial x}\right)+q_{2} \frac{\partial \omega^{(n)}}{\partial x}+q_{3} \omega^{(n)}=f\left(x, t, \omega^{(n-1)}+\zeta, \frac{\partial \omega^{(n-1)}}{\partial x}+\frac{\partial \zeta}{\partial x}\right), \\
\omega^{(n)}(x, 0)=0, \quad \omega^{(n)}(1, t)=0, \quad \int_{0}^{b} \omega^{(n)}(x, t) d x=0 .
\end{gathered}
$$

Theorem 2.8 implies, for fixed $n$, that each of problems (3.5) possesses a unique solution $\omega^{(n)}(x, t)$. Taking its difference for $n=k$ and $n=k+1$, it follows that

$$
\begin{gathered}
\frac{\partial z^{(k)}}{\partial t}-\frac{\partial}{\partial x}\left(q_{1} \frac{\partial z^{(k)}}{\partial x}\right)+q_{2} \frac{\partial z^{(k)}}{\partial x}+q_{3} z^{(k)}=\tilde{f}^{(k-1)}(x, t), \\
z^{(k)}(x, 0)=0, \quad z^{(k)}(1, t)=0, \quad \int_{0}^{b} z^{(k)}(x, t) d x=0
\end{gathered}
$$

where

$$
\begin{gathered}
z^{(k)}(x, t)=\omega^{(k+1)}(x, t)-\omega^{(k)}(x, t), \\
\tilde{f}^{(k-1)}(x, t)=f\left(x, t, \omega^{(k)}+\zeta, \frac{\partial \omega^{(k)}}{\partial x}+\frac{\partial \zeta}{\partial x}\right)-f\left(x, t, \omega^{(k-1)}+\zeta, \frac{\partial \omega^{(k-1)}}{\partial x}+\frac{\partial \zeta}{\partial x}\right) .
\end{gathered}
$$

\subsection{A priori estimates}

Theorem 3.1. Let Assumption 2.1 be fulfilled, let $f(x, t, 0,0) \in L^{2}\left(I, L_{\rho}^{2}(0,1)\right)$, and let $f(x, t, r, y)$ satisfy the Lipschitz condition, that is,

$$
\exists L>0:\left\|f\left(x, t, r_{1}, y_{1}\right)-f\left(x, t, r_{2}, y_{2}\right)\right\| \leq L\left(\left\|r_{1}-r_{2}\right\|+\left\|y_{1}-y_{2}\right\|\right) .
$$


Then

$$
\left\|z^{(k)}\right\|_{B} \leq c_{12}\left\|z^{(k-1)}\right\|_{B}, \quad k=1,2, \ldots
$$

where $c_{12}=L \sqrt{2 T c_{11}}$.

Proof. Integrating by parts $\int_{0}^{t}\left(\tilde{f}^{(k-1)}(\cdot, \tau), M z^{(k)}(\cdot, \tau)\right)_{L^{2}(0,1)} d \tau$ and proceeding as in the establishment of Theorem 2.6, we get

$$
\int_{0}^{t}\left\|\frac{\partial z^{(k)}(\cdot, \tau)}{\partial t}\right\|_{L_{\rho}^{2}(0,1)}^{2} d \tau+\left\|z^{(k)}(\cdot, t)\right\|_{H_{\rho}^{1}(0,1)}^{2} \leq c_{11} \int_{0}^{t}\left\|\tilde{f}^{(k-1)}(\cdot, \tau)\right\|_{L_{\rho}^{2}(0,1)}^{2} d \tau \text {, }
$$

where $c_{11}=4 \exp \left(c_{7} T\right) / \min \left(c_{0} / 2, b^{2} / 4\right)$.

Applying Lipschitz inequality to the right-hand side of (3.10), we obtain

$$
\begin{aligned}
& \int_{0}^{t}\left\|\frac{\partial z^{(k)}(\cdot, \tau)}{\partial t}\right\|_{L_{\rho}^{2}(0,1)}^{2} d \tau+\left\|z^{(k)}(\cdot, t)\right\|_{H_{\rho}^{1}(0,1)}^{2} \\
& \quad \leq 2 c_{11} L^{2}\left(\left\|z^{(k-1)}\right\|_{L^{2}\left(I, L_{\rho}^{2}(0,1)\right)}^{2}+\left\|\frac{\partial z^{(k-1)}}{\partial x}\right\|_{L^{2}\left(I, L_{\rho}^{2}(0,1)\right)}^{2}\right) \\
& \quad \leq 2 c_{11} L^{2}\left\|z^{(k-1)}\right\|_{L^{2}\left(I, H_{\rho}^{1}(0,1)\right)}^{2} \leq 2 c_{11} T L^{2}\left\|z^{(k-1)}\right\|_{C\left(I, H_{\rho}^{1}(0,1)\right)}^{2} \\
& \quad \leq 2 c_{11} T L^{2}\left\|z^{(k-1)}\right\|_{B}^{2} .
\end{aligned}
$$

The right-hand side of the above inequality being independent of $t$, we take the supremum with respect to $t$ in the left-hand side. It then follows that

$$
\left\|z^{(k)}\right\|_{B}^{2} \leq 2 c_{11} T L^{2}\left\|z^{(k-1)}\right\|_{B}^{2}
$$

from which we have estimate (3.9).

\subsection{Existence of the solution}

TheOREM 3.2. Let the hypotheses of Theorem 3.1 hold. If

$$
L<\frac{1}{\sqrt{2 T c_{11}}}
$$

then problem (3.4) admits a weak solution in the space $B$.

Proof. It is easy to see that if $c_{12}<1$, that is, $L<1 / \sqrt{2 T c_{11}}$, then the series $\sum_{k \geq 1} z^{(k)}(x, t)$ converges. Consequently, the sequence $\left\{\omega^{(n)}\right\}_{n \in \mathbb{N}}$ defined by

$$
\omega^{(n)}(x, t)=\sum_{k=0}^{n-1}\left(\omega^{(k+1)}(x, t)-\omega^{(k)}(x, t)\right)+\omega^{(0)}(x, t), \quad n=1,2, \ldots
$$


converges, in the norm of space $B$, to $\widetilde{\omega}(x, t)$. To prove that this function is a weak solution of problem (3.4), we proceed exactly as in the proof of Theorem 2.8 .

3.4. Uniqueness of the solution. In this section, we first establish an a priori estimate. The uniqueness of the solution is then a direct corollary of it.

Theorem 3.3. Under the hypotheses of Theorem 3.1, assume that $\omega_{1}$ and $\omega_{2}$ are two solutions in B of problem (3.4). Then

$$
\left\|\omega_{1}-\omega_{2}\right\|_{B} \leq c_{12}\left\|\omega_{1}-\omega_{2}\right\|_{B}
$$

Proof. Let $\omega(x, t)=\omega_{1}(x, t)-\omega_{2}(x, t),(x, t) \in \bar{\Omega}$. Then $\omega(x, t)$ satisfies

$$
\begin{gathered}
\frac{\partial \omega}{\partial t}-\frac{\partial}{\partial x}\left(q_{1}(x, t) \frac{\partial \omega}{\partial x}\right)+q_{2}(x, t) \frac{\partial \omega}{\partial x}+q_{3}(x, t) \omega=\tilde{f}(x, t), \\
\omega(x, 0)=0, \quad \omega(1, t)=0, \quad \int_{0}^{b} \omega(x, t) d x=0,
\end{gathered}
$$

where

$$
\tilde{f}(x, t)=f\left(x, t, \omega_{1}+\zeta, \frac{\partial \omega_{1}}{\partial x}+\frac{\partial \zeta}{\partial x}\right)-f\left(x, t, \omega_{2}+\zeta, \frac{\partial \omega_{2}}{\partial x}+\frac{\partial \zeta}{\partial x}\right) .
$$

Performing similar calculations to that for Theorem 3.1, we obtain

$$
\|\omega\|_{B} \leq c_{12}\|\omega\|_{B}
$$

From estimate (3.15), we conclude that

$$
\left(1-c_{12}\right)\left\|\omega_{1}-\omega_{2}\right\|_{B} \leq 0 .
$$

Since $c_{12}<1$, then $\left\|\omega_{1}-\omega_{2}\right\|_{B}=0$, that is, $\omega_{1}(x, t) \equiv \omega_{2}(x, t)$, for all $(x, t) \in \Omega$, from which we have the following corollary.

Corollary 3.4. Suppose that the hypotheses of Theorem 3.1 are satisfied, then the solution of problem (3.4) is unique.

\section{References}

[1] N.-E. Benouar and N. I. Yurchuk, A mixed problem with an integral condition for parabolic equations with a Bessel operator, Differentsial'nye Uravneniya 27 (1991), no. 12, 2094-2098.

[2] A. Bouziani, Mixed problem with boundary integral conditions for a certain parabolic equation, J. Appl. Math. Stochastic Anal. 9 (1996), no. 3, 323-330.

[3] On a class of parabolic equations with a nonlocal boundary condition, Acad. Roy. Belg. Bull. Cl. Sci. (6) 10 (1999), no. 1-6, 61-77. 
[4] On the solvability of a class of singular parabolic equations with nonlocal boundary conditions in nonclassical function spaces, Int. J. Math. Math. Sci. 30 (2002), no. 7, 435-447.

[5] - On the solvability of parabolic and hyperbolic problems with a boundary integral condition, Int. J. Math. Math. Sci. 31 (2002), no. 4, 201-213.

[6] - On three-point boundary value problem with a weighted integral condition for a class of singular parabolic equations, Abstr. Appl. Anal. 7 (2002), no. 10, 517530.

[7] A. Bouziani and N.-E. Benouar, Sur un problème mixte avec uniquement des conditions aux limites intégrales pour une classe d'équations paraboliques [A mixed problem with only integral boundary conditions for a class of parabolic equations], Maghreb Math. Rev. 9 (2000), no. 1-2, 55-70 (French).

[8] J. R. Cannon, The solution of the heat equation subject to the specification of energy, Quart. Appl. Math. 21 (1963), 155-160.

[9] The One-Dimensional Heat Equation, Encyclopedia of Mathematics and Its Applications, vol. 23, Addison-Wesley, Massachusetts, 1984.

[10] J. R. Cannon, S. Pérez Esteva, and J. van der Hoek, A Galerkin procedure for the diffusion equation subject to the specification of mass, SIAM J. Numer. Anal. 24 (1987), no. 3, 499-515.

[11] J. R. Cannon and J. van der Hoek, The classical solution of the one-dimensional twophase Stefan problem with energy specification, Ann. Mat. Pura Appl. (4) 130 (1982), 385-398.

[12] , Diffusion subject to the specification of mass, J. Math. Anal. Appl. 115 (1986), no. 2, 517-529.

[13] V. Capasso and $\mathrm{K}$. Kunisch, A reaction-diffusion system arising in modelling manenvironment diseases, Quart. Appl. Math. 46 (1988), no. 3, 431-450.

[14] Y. S. Choi and K.-Y. Chan, A parabolic equation with nonlocal boundary conditions arising from electrochemistry, Nonlinear Anal. 18 (1992), no. 4, 317-331.

[15] W. A. Day, Extensions of a property of the heat equation to linear thermoelasticity and other theories, Quart. Appl. Math. 40 (1982), no. 3, 319-330.

[16] N. I. Ionkin, The solution of a certain boundary value problem of the theory of heat conduction with a nonclassical boundary condition, Differentsial'nye Uravneniya 13 (1977), no. 2, 294-304.

[17] L. I. Kamynin, A boundary-value problem in the theory of heat conduction with nonclassical boundary conditions, Ž. Vyčisl. Mat. i Mat. Fiz. 4 (1964), 1006-1024.

[18] P. Shi and M. Shillor, On design of contact patterns in one-dimensional thermoelasticity, Theoretical Aspects of Industrial Design (Wright-Patterson Air Force Base, OH, 1990), SIAM, Philadelphia, 1992, pp. 76-82.

[19] N. I. Yurchuk, A mixed problem with an integral condition for some parabolic equations, Differentsial'nye Uravneniya 22 (1986), no. 12, 2117-2126.

Abdelfatah Bouziani: Department of Mathematics, The Larbi Ben M'hidi University Centre, Oum El Bouagui 04000, Algeria

Current address: The Abdus Salam International Centre for Theoretical Physics, Strada Costiera 11, 34100 Trieste, Italy 


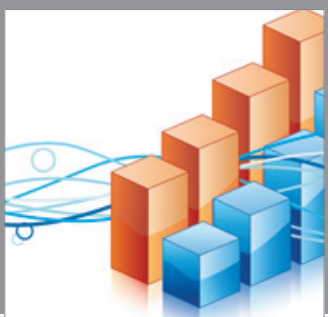

Advances in

Operations Research

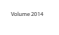

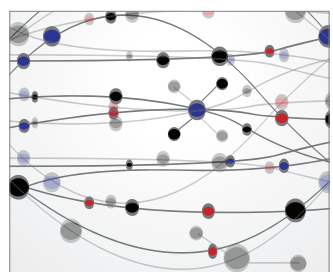

\section{The Scientific} World Journal
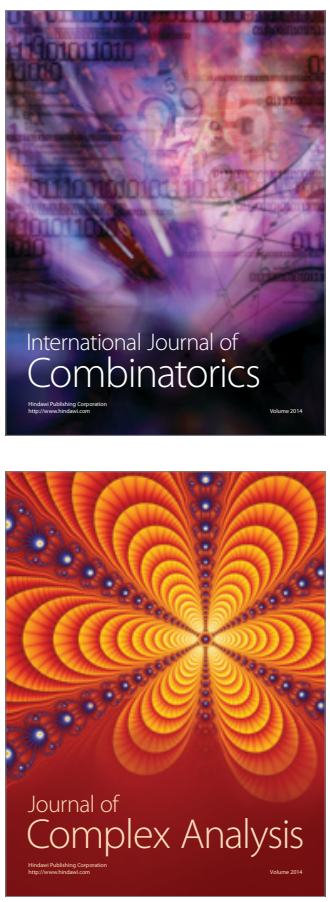

International Journal of

Mathematics and

Mathematical

Sciences
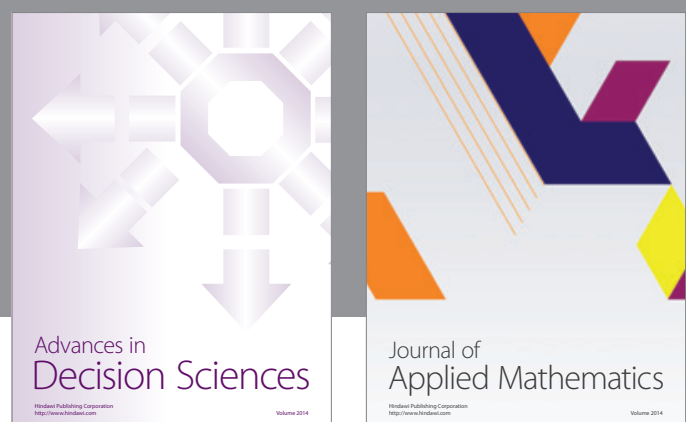

Journal of

Applied Mathematics
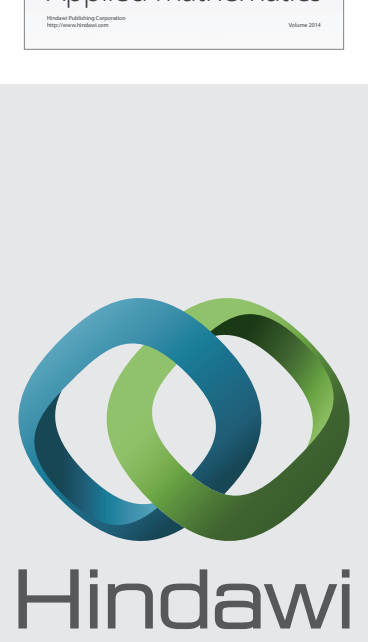

Submit your manuscripts at http://www.hindawi.com
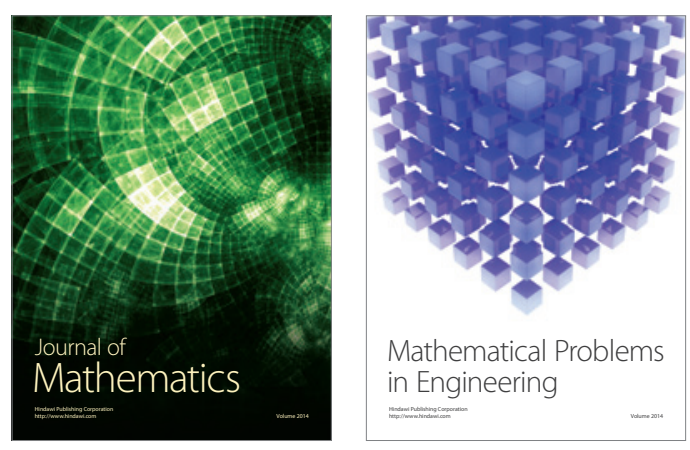

Mathematical Problems in Engineering
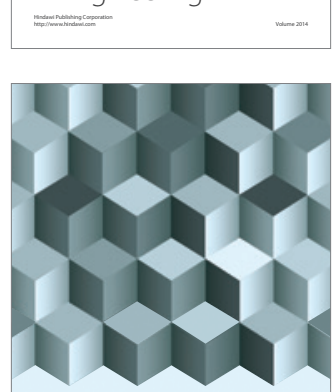

Journal of

Function Spaces
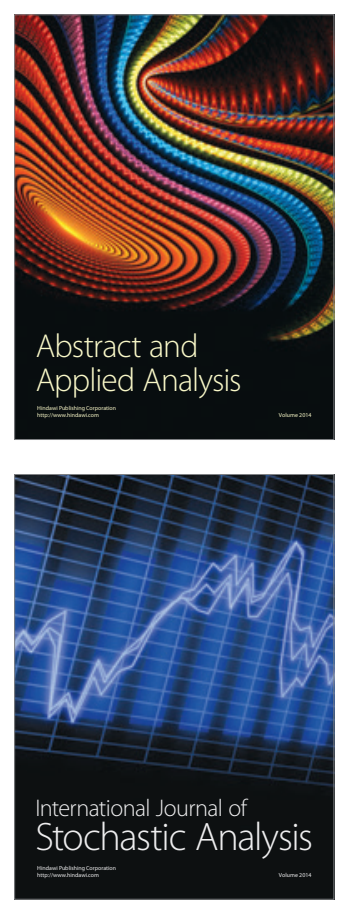

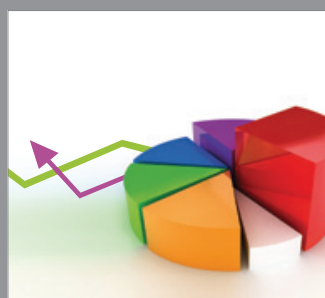

ournal of

Probability and Statistics

Promensencen
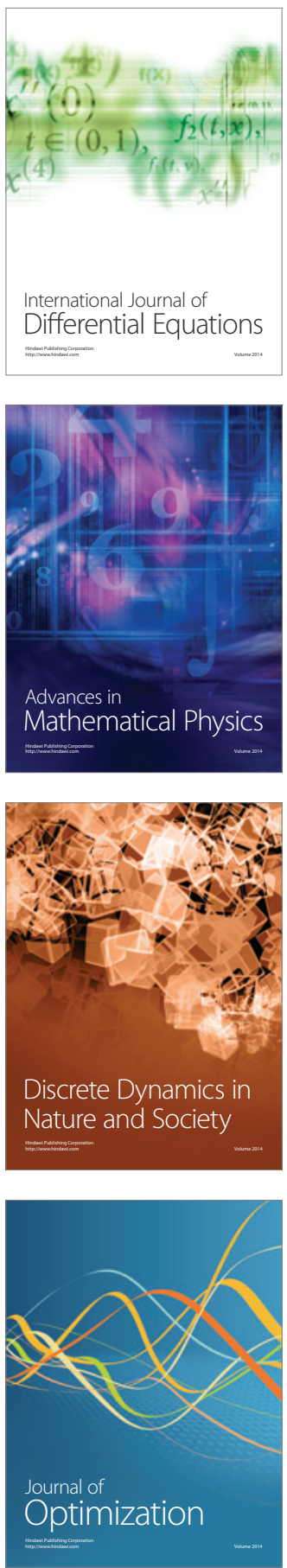